\title{
PRÁCTICAS PEDAGÓGICAS INCLUSIVAS DESDE EL DISEÑO UNIVERSAL DE APRENDIZAJE Y PLAN INDIVIDUAL DE AJUSTE RAZONABLE
}

\section{INCLUSIVE EDUCATIONAL PRACTICES FROM UNIVERSAL DESIGN FOR LEARNING AND INDIVIDUAL PLAN OF REASONABLE ADJUSTMENT}

Luz Aide Figueroa Zapata

Ifiguer5@uniminuto.edu.co

Corporación Universitaria Minuto de Dios - UNIMINUTO

Bogotá-Colombia

María Soledad Ospina García mospinagarc@uniminuto.edu.co Corporación Universitaria Minuto de Dios- UNIMINUTO Bogotá- Colombia

Jennifer Tuberquia Tabera jtuberquiat@uniminuto.edu.co Corporación Universitaria Minuto de Dios- UNIMINUTO Bogotá-Colombia

Fotografia de portada y Diagramación Sindy Catherine Charcas Ibarra 


\section{RESUMEN}

La obligación de las instituciones educativas es impartir educación de calidad a todos los niños sin excepción y procurar porque cada uno desarrolle sus dimensiones y las competencias mínimas para desenvolverse en sociedad, por lo tanto, el mandato constitucional de inclusión, debe equipararse a los recursos que brinda el estado y a las didácticas del docente. Este artículo pretende recoger los conceptos y caracterizar las prácticas pedagógicas que circundan alrededor de la implementación del DUA (Diseño Universal de Aprendizaje) y los PIAR (Planes Integrados de Ajustes Razonables) en las aulas regulares, así como dar a conocer los imaginarios y las diferentes prácticas que utilizan algunos docentes en sus centros educativos. Con una metodología mixta utilizando rastreo en bases de datos, un cuestionario debidamente validado y aplicado a 30 docentes en 20 centros infantiles de Medellín y un grupo focal. Las conclusiones determinan los conceptos y prácticas propuestas por el DUA y la implementación de los PIAR; Los datos evidencian que el modelo de inclusión es apenas una experimentación de actividades individualizadas, poco se pone en práctica el DUA, y no se evidencia que se diseñen Planes Individuales de Ajuste Razonables -PIAR-. Para concluir se puede determinar que entre la Ley y su aplicación aún hay una brecha muy amplia, y que el docente en ejercicio poco se ocupa de cerrarla a pesar del mandato constitucional.

Palabras clave: inclusión, diseño universal de aprendizaje, PIAR, prácticas pedagógicas, educación.

\section{ABSTRACT}

The educational institutions have an obligation to provide quality education to all children without exception; also they must ensure that each one of these develops its dimensions and the minimum competencies to function in society, therefore, the constitutional mandate of inclusion must be equated with the resources that provides the state and the teacher's teaching. This article aims to collect the concepts and characterize the pedagogical practices surrounding the implementation of the DUA (Universal Design of Learning) and the PIAR (Integrated Plans of Reasonable Adjustments) in the regular classrooms, as well as to make known the imaginaries and the different practices that some teachers use in their educational centers. A mixed methodology using database tracking was carried out, also a questionnaire validated and applied to 30 teachers in 20 children's centers in Medellín and a focus group. The conclusions determine the concepts and practices proposed by the DUA and the implementation of the PIAR; The data show that the inclusion model is just an experimentation of individualized activities; the DUA is put into practice to a limited extent, and there is no evidence that Individual Plans of Reasonable Adjustment -PIAR- are designed. To conclude, it can be determined that between the Law and its application there is still a very wide gap, the teacher is not aware about it, despite the constitutional mandate.

Keywords: inclusion, universal learning design, Integrated Plans of Reasonable Adjustments, pedagogical practices, education. 


\section{INTRODUCCIÓN}

La inclusión social en Colombia es un tema que apenas empieza a tener importancia en las diferentes esferas sociales, pero que está presente como un problema de concepción y de convivencia común en la escuela. Las instituciones educativas del país que atienden primera infancia están obligadas al cumplimiento de la Ley, por lo tanto, sus docentes y toda la comunidad educativa deberá implementar estrategias necesarias para que la escuela sea un espacio inclusivo, sin más barreras que las que cada persona con discapacidades o Necesidades Educativas Especiales imponga a nivel personal. La inquietud en esta reflexión radica en el proceso de enseñanza y las prácticas pedagógicas que los docentes deben emplear para atender las necesidades de cada uno de los niños que tienen barreras para el aprendizaje y que ingresan a la escuela por derecho propio contemplado en la Ley Estatutaria 1618 de 2013, Ley de Inclusión educativa, en cuya aplicación los docentes no tienen ni capacitación, ni suficientes herramientas de manejo pedagógico para garantizar el éxito de la inclusión. Al establecer que las instituciones educativas tienen que ser espacios inclusivos, (Ley 1618 de 2013) desde la convivencia, el sistema de matrículas, enseñanza y evaluación, desarrollo de competencias comunicativas, emocionales y científicas, es necesario profundizar sobre los elementos pedagógicos que hacen posible la inclusión y entenderla como la forma de alcanzar los objetivos propuestos en la misma Ley General de Educación, II 5 de 1994; en desde la escuela, la familia y la sociedad, es darle una mirada sobre las prácticas pedagógicas que circulan al interior de la escuela. De esta manera y luego de haber realizado un primer acercamiento al quehacer de la escuela desde la investigación realizada por Figueroa (2017) sobre el impacto de la Ley de Inclusión en la ciudad de Medellín, Colombia, se concluye que ni la escuela, ni los maestros, ni el mismo sistema están preparando para afrontar el reto. La inclusión no se puede determinar como una simple incursión a la escuela donde todos están al tanto de las falencias de los sujetos involucrados, es, podría decirse, un proyecto que inicia en los primeros años escolares y que termina con el desarrollo de un proyecto de vida productivo y satisfactorio en el marco de la ciencia, la cultura y el desempeño social. Así lo planteó Salmi, J. (2016. P 15) "Inclusión es asegurarse de que todos los grupos en una sociedad tengan la oportunidad no solamente de acceder a la educación, sino también de terminar exitosamente" a la vez afirma que es un error cuando solo se da importancia al acceso al sistema escolar pero que una vez adentro los procesos no son muy claros ni propicios para el desarrollo de las diferentes competencias.

\section{INTRODUCCIÓN}

\section{Apuntes conceptuales}

\section{I.I. Antecedentes: Inclusión versus integración}

Hasta hace algunas décadas era común encontrar tratados e investigaciones respecto a la diversidad étnica, a la condición social y económica de las sociedades y eso se convertía en motivo para hablar de prácticas inclusivas o excluyentes, tanto en la escuela como en el campo laboral, es decir que la discriminación se había mirado solo desde este ángulo, y la escuela no estaba pensada para casos como la discriminación escolar por razones de inhabilidades motrices, psicológicas o mentales- cognitivas. Para tal caso, solo algunos centros especializados atendían una minoría, que por lo general eran de estratos económicos altos, pero no era notable que se excluyera una alta población con NEE que no tenían atención. Para el caso que interesa en esta investigación, se podría citar el trabajo de Carlos A. Viáfara López en: Diferencias raciales en el logro educativo y status ocupacional en el primer empleo, en la ciudad de Cali (Colombia) 2008, el investigador establece que a través de su estudio confirma las premisas establecidas en el modelo deBlau y Duncan (1967: 163-205), comentadas por Viáfara:

Por un lado, se corroboró la importancia significativa de los orígenes sociales en el logro educativo ya que un buen antecedente familiar suele estar asociado y participar con mayores recursos materiales, a sus allegados en condiciones de discapacidad y garantizar el éxito en las carreras educativas de los individuos, así como influir en las aspiraciones, características de personalidad, valores y la transmisión de las habilidades en pobreza y exclusión social. Por otro lado, el efecto del logro educativo, y no el efecto de los orígenes sociales, es preponderante en el status ocupacional en el primer empleo. (2008).

Para poder plantear un amplio concepto de cómo se han tratado las discapacidades humanas, es necesario remitirse a las civilizaciones antiguas. Vale la pena citar desde Mesopotamia cómo la medicina de la época clasificaba tres practicas principales con relación a la salud: la adivinación, los conjuros y exorcismos; y prácticas médicas sencillas como vendajes y pequeñas cirugías Duchan (20II). Sin embargo, se observa en los registros históricos que mantenían dos tipos de discapacidades: Discapacidad Leve que en algunos casos se les daba apoyo y los empleaban atendiendo las necesidades de los dioses. Discapacidad Severa: se consideraban a menudo peligrosos para la sociedad y era para ellos un castigo.

Otra de las civilizaciones que se preocuparon por la salud fueron los egipcios quienes se destacaron por hacer grandes investigaciones con personas fallecidas, se hacían diagnósticos a través de la observación cuidadosa pero no hay muchos registros de las discapacidades y no tuvieron un lugar en la sociedad, aunque guardaban respeto por las personas con discapacidad Hernández (2010). Por su parte, la sociedad hebrea se refería a la discapacidad como una "marca del pecado", en Levítico (2I: 17- 2I) se señala que, "Si alguno de tus descendientes tiene algún defecto físico, no podrá acercarse a mi altar para presentarme las ofrendas"; las personas con discapacidades se empleaban para el cuidado de las ovejas, el comercio y oficios informales. Di Nasso (2010, p. 9 y I0)

Entre los griegos el culto a la belleza y la perfección física pudo haber influenciado en situaciones de discriminación económica y social, consideraban a las personas con discapacidad en el mismo rango de los esclavos y criminales y los expulsaban de la comunidad. Según lo menciona Di Nasso (2010, p. 9 y 10), así mismo en los pueblos de Asia y África sostenían diversas prácticas discriminatorias. En la India los niños y niñas con discapacidad eran abandonados en el bosque o arrojados al río Ganges donde purificarían sus pecados y 
Prácticas pedagógicas inclusivas desde el diseño universal de aprendizaje y plan individual de ajuste razonable

alcanzarían la perfección en la siguiente vida. Por otro lado, en la Antigua China se acudía a terapias medicinales como la cinoterapia y los masajes para tratar ayudar a las personas que padecieran alguna discapacidad motriz y había consideración y respeto para ellas como lo proponía Confucio (55I- 479 AC) por considerarlas personas “débiles”.

Por su parte los Romanos, consideraban la discapacidad como un defecto que debía ser eliminado, y quienes la padecían sufrían de discriminación, burlas o se sometían a escarnio público, por lo que el emperador Claudio ( I0-54 DC) sufrió a causa de su fea apariencia física y falta de salud, (Gutiérrez,p. 32, 1997)

Para la edad media la situación no era muy distinta, los discriminados veían en la religión el escape a sus preocupaciones y la posibilidad de tener una esperanza para una mejor vida, aunque la situación social de las poblaciones más desfavorecidas endureció, pues la concepción de que de las deformidades o discapacidades en el hombre eran producto del pecado y debían ser castigadas. Di Nasso,(2010, p I I). Lejos no estuvieron los siglos siguientes, con una leve consideración luego de la proclamación de los Derechos Fundamentales resultado de la Revolución Francesa en los inicios del siglo XIX.

Ante este panorama, Warnock (1978), planteó el concepto de "Integración educativa" para los niños y jóvenes con NEE donde se señalaron los principios básicos del derecho que debía ser el mismo para todos, y que se vulneraba. A través de este concepto se estimó que "La educación debía iniciar inmediatamente después del diagnóstico de la deficiencia y asegurar la suficiente formación básica y continua de los docentes que atenderían este tipo de población". De esta manera, los europeos también hicieron énfasis en la necesidad del acompañamiento familiar para una mejor adaptación de los estudiantes con NEE dentro de las aulas regulares y de la escuela, considerando esta como marco normalizado y normalizador de la educación, y convirtiendo la escuela en un centro de recursos especializados y de apoyo.

Adicionalmente, hasta hace muy poco, las políticas de educación para estas personas eran bastante reducidas o nulas, especialmente si pertenecían a estratos sociales bajos, o por el desconocimiento de las habilidades personales de los "niños especiales". Es de considerar que en Francia se empieza a dar los primeros pasos en las postrimerías del siglo XIX, en la atención personal con los estudios y el trabajo realizado por Jean Marc Gaspar d'Itard en el instituto para sordomudos en París, y quien fue considerado el precursor de la educación especial con su famoso caso del niño salvaje de Aveyron o la reeducación de Victor de L`Aveyron.

El concepto de deficiencia o especiales se mantiene hasta finales del siglo $X X$ con los estudios de Marchesi $y$ Martin en 1990, Castejón y Lavas (2000 p. I3 - 20) ya que la forma de determinar el tipo de trastorno era a través de los dos tipos de test de inteligencia elaborados por Galton y Binet cuyos resultados permitían separar a los niños y niñas por las características de sus deficiencias y de esta manera se remitía al centro educativo de su especialidad. A lo anterior se aprecia el interés de la psicología conductista que introduce el concepto de la influencia de los factores ambientales para explicar la conducta de deficiencia intelectual asumiéndola como el pobre aprendizaje desde su entorno, o aprendizajes mal dirigidos y poco motivados. Para lo anterior, Marchesi y Martin en (1990) siguen sosteniendo la idea de una educación separada para este tipo de personas.

Nuevamente Warnock (1978), plantea un concepto bastante apreciable para el sistema educativo "Un niño o niña con NEE es aquel que le cuesta aprender lo que otros al mismo ritmo o con la misma profundidad". Desde este postulado se señala que los principios educativos son iguales para todos, pero que es necesario ampliar los conceptos, didácticas y procesos de enseñanza - aprendizaje para que sean incluyentes, y no se mire como un caso independiente de educación donde el docente regular se deba extra actuar para poder atender la población NEE.

Para finales del siglo $X X$, Colombia también hace sus esfuerzos por buscar la equidad e incluir a las personas con limitaciones en el sistema escolar y se crean algunas escuelas para niños llamados “especiales”, así que la tarea es que la escuela y los entes involucrados busquen metodologías inclusivas como un sistema cooperativo, constructivo y reflexivo donde toda la sociedad participe, Arnáiz (2003). Por lo anterior, es importante "concebir el aula como un lugar de intercambio de experiencias y de cultura, de implicación, autoconocimiento, autonomía, comunicación y socialización, un espacio abierto en el que se da cabida al conjunto de diversidades que presenta el alumnado Es fundamentalmente un espacio de participación activa, de interacción y diálogo en el que la aceptación, el cuestionamiento, el rechazo o la asunción de procesos orientarán la enseñanza-aprendizaje y determinarán también el clima cultural y social de la misma" ( Moliner, 2017. p.26)

Es común que en el ámbito educativo se tienda a confundir el término inclusión con el de integración de niños con barreras de aprendizaje al aula regular, y aunque desde el ingreso de estos al sistema educativo comparten los mismos espacios físicos, no se podría afirmar que eso implique que están o se sientan incluidos dentro de los procesos de enseñanza aprendizaje que los lleven a desarrollar las competencias mínimas desde lo procedimental, lo actitudinal y lo cognitivo. Al respecto, Blanco, R. (2009, p. 83-99) establece que éste es un paradigma ya que las adaptaciones se dan con muchas restricciones, desde diferentes perspectivas, a veces no fáciles, que van desde el currículo, las normas, las áreas en las cuales se pretende desarrollar competencias, en las didácticas que tienen los docentes, entre otras más, y que de alguna manera hacen exclusiones dentro de las diferentes limitaciones que presentan en los niños que llegan al sistema.

Es de público conocimiento que se ha legislado desde los estamentos gubernamentales y se han determinado políticas de inclusión educativa que tienen como fin materializar las prácticas pedagógicas de los docentes, pero es el maestro quien vivencia la diversidad en su aula de clase, conoce las necesidades e intereses de sus estudiantes, es el principal orientador del proceso educativo de los niños y a través de las diferentes prácticas, es quien propiciará una verdadera 
inclusión tanto desde lo cognitivo como desde lo social. Al respecto, Ocampo (2015. p. 37) manifiesta que es necesario "Comprender el funcionamiento de la mecánica de la exclusión y su relación con la inclusión, establece un "diálogo polifonal", citando a Fornet-Betancourt (1999), debido a que permite la emergencia de nuevas voces, condiciones y procesos que explican la inclusión como un proceso dinámico, cuyo grado de incidencia afecta a la igualdad de posiciones que condicionan la vida social de todos los ciudadanos", pero además antepone que puede trascender como si fuera la "imposición de un modelo tradicional de educación especial, limitando su potencial de transformación" (p.37) en cuyo caso este diálogo de heterogeneidades puede facilitar la validación y participación de diversos actores como parte de un mismo proyecto ético-político- histórico-cultural dentro del macro proyecto educativo, concluye Ocampo.

\subsection{Barreras para Aprendizaje y desarrollo de competencias}

La Declaración Universal de los Derechos Humanos (1948) en el capítulo I refiere a los derechos que poseen todas las personas y el Articulo 19 de la Convención Internacional de los Derechos del impedido (ONU, 2009) ratifica que todas las personas con discapacidad son titulares de los mismos derechos fundamentales que el resto de los ciudadanos, que son libres e iguales en dignidad y derechos. A fin de alcanzar esta meta, todas las comunidades deben celebrar la diversidad en el seno de sí mismas, $y$ asegurar que las personas con discapacidad puedan disfrutar de todos los Derechos Humanos: civiles, políticos, sociales, económicos y culturales reconocidos por las distintas Convenciones internacionales. Con lo anterior, queda claro que es una obligación social la inserción de niños con barreras para el aprendizaje al sistema escolar y poder propiciar espacios que les permitan desarrollar sus capacidades y habilidades de tal manera que se puedan desenvolver de manera autónoma en lo social y lo individual; de ser posible que el individuo desarrolle competencias con miras a alcanzar el Desarrollo Humano a través de un proyecto de vida pertinente y posible.

Así mismo el Artículo 20, numeral I, sobre Educación de la convención mencionada (ONU, 2006), sobre educación, establece que "Los Estados Partes reconocen el derecho de las personas con discapacidad a la educación con miras a hacer efectivo este derecho sin discriminación y sobre la base de la igualdad de oportunidades". Y por lo tanto el Estado deberá legislar sobre el derecho a una educación inclusiva a todos los niveles y a lo largo de la vida que se pueda establecer, en los términos de Nussbaum (20II) desde el desarrollo de las capacidades del ser humano que deben ir de la mano del respeto por la dignidad de cada individuo, derecho que requiere del apoyo político y de la implementación de leyes equitativas y permanentes para que no caigan en el olvido del deber ser. Por tal razón, Egler (2017, p. 20) se refiere a que "La formación inicial y continua de los profesores tanto de enseñanza corriente como especial, necesita reorientarse según los principios de la educación inclusiva, para reemplazar de una vez los restos del modelo segregacionista aún presente en las prácticas educativas y ser transparentes en el discurso que vincula la Atención Educativa Especializada (AEE) con la rehabilitación y las prácticas de enseñanza diferenciada según la condición de discapacidad de algunos estudiantes". Evidentemente los docentes son los que más deben estar preocupados ante esta avalancha de situaciones dentro de las aulas regulares, máxime si la capacitación o los recursos por parte del estado no son suficientes, o talvez, la disposición y vocación de servicio no son altos y la Ley presiona para ejecutar como una tarea obligada.

\section{I.3. Diversidad Cultural}

La situación de diversidad cultural y segregación es un problema creciente en el mundo, "La segregación racial, las diferentes corrientes religiosas y políticas aún no se acaban, a pesar de que el desarrollo de la humanidad no parece tener límites en otros campos como las ciencias, las comunicaciones o las tecnologías" (De Soussa, 2010. p. 48), en esta perspectiva, la escuela es un universo de diferencias latentes entre sus miembros, y por tal razón la pedagogía direcciona sus métodos tendientes a crear espacios donde el encuentro de diferentes culturas, pensamientos y formas de actuar tengan cabida. El respeto por la singularidad está en el plano de las tareas del maestro, aspecto que no es fácil, que además no tiene un manual para aplicar en cada situación y, por otro lado, esa singularidad tiene ingredientes como la desmotivación, el poco acompañamiento familiar, barreras de salud, que en muchos casos es graves y se convierten en un reto para el maestro. Por esta razón, el modelo educativo tradicional es selectivo y excluyente, la diversidad en las aulas es apenas una propuesta desde la legislación mundial en derechos humanos, la calidad educativa se mide por el rendimiento estándar que un estudiante obtenga en pruebas nacionales e internacionales y no como el ideal planteado por Gimeno,

Al ser, la diversidad entre los seres humanos o entre grupos de ellos y la singularidad individual entre sujetos, condiciones de nuestra naturaleza, el tema de las diferencias aparece como una dimensión que está siempre presente en cualquier problema que abordemos en educación" (2006.p. 17).

Lo que da a entender, que, para atender cualquier situación educativa, nunca se debe desconocer la diversidad, pues es a través de su reconocimiento como se puede lograr el desarrollo humano.

\section{Marco teórico}

\section{I. Prácticas pedagógicas}

Se definen como todas aquellas experiencias didácticas, metodológicas fijas o espontaneas dirigidas por un tutor o maestro para desarrollar una actividad educativa tendiente a que los participantes, en este caso estudiantes, logren integrar sus conocimientos previos a los producidos por el acercamiento a las ciencias, de tal manera que el resultado sea un aprendizaje significativo y el afianzamiento de unas competencias establecidas para cada actividad de su vida. En el caso de las prácticas pedagógicas inclusivas se deben tener en cuenta ciertas características como la adaptación de las mismas a las necesidades de los estudiantes, y deben posibilitar el desarrollo autónomo y respetuoso desde la diversidad, como 
Prácticas pedagógicas inclusivas desde el diseño universal de aprendizaje y plan individual de ajuste razonable

lo expresa Blanco,

En la inclusión, por el contrario, el foco de atención es transformar los sistemas educativos y las escuelas para que sean capaces de acoger a todos los estudiantes de la comunidad y dar respuesta a la diversidad de necesidades de aprendizaje que son fruto de su procedencia social y cultural y sus características individuales en cuanto a capacidades, motivaciones, estilos y ritmos de aprendizaje. (20I I, pág. 3)

De lo anterior se deduce que cada práctica de aula debe tener un propósito claro y preciso al momento de aplicarla, debe ser planeada de acuerdo a los ritmos, necesidades y aptitudes de los niños con barreras, y su culminación no se define en el tiempo sino en la capacidad de utilizar tales aprendizajes para desenvolverse en ciertos contextos; sean sociales, personales, o donde se requiera de una habilidad específica. Del mismo modo, estos procesos de aprendizaje y su resultado son diferentes para cada individuo, pues cada participante con barreras es un caso diferente, son individualidades, motivaciones muy personales y procesos de acompañamiento, tan distintos como ellos mismos, de tal manera que "es el sistema educativo el que debe cambiar para contemplar la diversidad en nuestras aulas y no al revés" (López. M, 20I I I. p.40).

Otras concepciones apuntan a definir la práctica como acciones pedagógicas productoras de sujetos en una mediación científica experimental en la cual el ser pedagógico es un individuo histórico que hace inmersión en la complejidad de un universo sociocultural, con una perspectiva más amplia que lo va asumiendo dentro de unos modelos socio afectivos de su entorno educativo y vivencial, de articulaciones posibles, educador - educando que legitiman su propio ser, como pilar en la pedagogía. (Zaccagnini, 2008)

Al profundizar en las didácticas y prácticas empleadas dentro de las aulas inclusivas, es necesario que los agentes educativos tengan un conocimiento amplio del Diseño Universal de Aprendizaje- DUA y que a través de él realicen las adecuaciones pertinentes del currículo, donde la flexibilización sea acorde al ritmo de los aprendizajes, pero teniendo muy en claro las metas consistentes en desarrollar las competencias mínimas frente a las tareas propuestas, (Arnaiz. 20I I).

\subsection{Calidad de vida en la educación inclusiva}

Podría afirmarse que este es una de las metas que se empiezan a lograr en el corto plazo en los modelos de educación inclusiva, pues desde el mismo inicio de la escolarización el niño comienza a sentirse participe de su proceso de socialización, allí empieza a desempeñarse como ser autónomo que busca construir su propio mundo en un contexto diferente a su hogar. Sin embargo, la cotidianidad de la escuela puede ser cruel si el centro educativo no ha implementado rutas de sensibilización con los alumnos regulares quienes pueden convertirse en sus verdugos.

El panorama cambia en la escuela cuando "La discapacidad deja de centrarse en la persona como núcleo de la intervención y objeto del concepto, para centrarse en el contexto como responsable de las posibilidades de participación, autonomía y normalización de su vida" (Muntaner 2013.p.37) en la American Association on Intellectual and Developmental Disabilities (AAIDD 2012). Desde esta perspectiva, la discapacidad pasa a ser una condición limitada del individuo focalizada solo en las deficiencias y limitaciones, y propone ser mirada como la relación entre las capacidades de la persona y el contexto en el que ha de desenvolverse; teniendo como principio mejorar las condiciones del entorno donde el individuo tiene el control de sus actuaciones sobre su discapacidad que ya no es una condición sino un estado o forma de funcionamiento, (Muntaner 2013.p.37)

Ante lo anterior, Nussbaum (2000) plantea el desarrollo humano desde el enfoque de las capacidades, y determina que los elementos más importantes de la calidad de vida de las personas son plurales, de tal manera que Gough (2007) las presenta en una lista de diez capacidades funcionales que los seres deberán desarrollar para alcanzar una vida digna. Una de ellas es la "Vida, en la cual los individuos deberán ser capaces de vivir una vida humana de duración normal hasta su fin, sin morir prematuramente o antes de que la vida se reduzca a algo que no merezca la pena vivir" (p. I82), pero adicional a ella menciona la salud y la integridad corporal con lo que se tiene la oportunidad de disfrutar de la libertad en lo amplio de su sentido. Así mismo incluye los sentidos, la imaginación y el pensamiento que van de la mano con la razón práctica en la que se crea la reflexión crítica, autónoma respecto de la planificación de la vida para ser compartida con otros en el compromiso social de que sean tratados como seres dignos que le otorga tal filiación. Eglér (2017) señala que "con todas las limitaciones que estos estudiantes puedan tener, necesitan socializar con niños normales ...donde van a tener todas las oportunidades de desarrollar potencialidades que tal vez no tendría fuera de ese ambiente" (p.15), y por supuesto hace parte de la integralidad del desarrollo pues no se tratará de aislarlo o darle un proceso de enseñanza de bajo conocimiento, de menor calidad, la cercanía con sus pares permitirá reconocer la escuela regular como una posibilidad para que "el estudiante con discapacidad se desenvuelva más de lo que lo haría en una escuela especial y que los profesores del aula regular necesitan contar con una formación en educación inclusiva que modifique su visión con respecto a la enseñanza y al aprendizaje” Eglér (2017p. 18).

Para las tres últimas capacidades funcionales, Gough (2007) parafraseando a Nussbaum, ratifica la necesidad de relacionarse con otras especies, es decir comprometerse con la biodiversidad además de desarrollar el ser político y participativo. Es muy interesante como destaca también la capacidad de jugar, de reír y disfrutar del ocio como fuente de goce personal. Finalmente, se resalta en este concepto de calidad de vida, en el concepto de dignidad humana y desarrollo humano concibe "a cada persona como un fin en sí misma y no se pregunta solamente por el bienestar total o medio, sino también por las oportunidades disponibles para cada ser humano; por tal razón, dicho en foque, se centran en ‘la elección o en la libertad”'. (Nussbaum, 2012.p.45). 


\section{Método}

El proyecto de investigativo consta de dos partes: la conceptualización de las implicaciones del DUA y los PIAR a través de diferentes experiencias y de la literatura existente, se enmarcó dentro del enfoque cualitativo, el cual permite acercarse a la realidad desde las dimensiones subjetivas y desde allí aprehenderla, sin perder la objetividad y rigor en la investigación del fenómeno que se está estudiando, puesto que la "naturaleza de la realidad social es objetiva y subjetiva a la vez" (Bonilla, 2005, p. 53) que además, se da relevancia a la "capacidad humana para percibir, explorar e interpretar la realidad" (Bonilla, 2005, p. 132); de tal manera que los comportamientos, actitudes, percepciones y vivencias in situ de los sujetos investigados, fueron insumos de gran valor en el análisis de la información. También se tuvo en cuenta el diseño de tipo etnográfico en el cual Hernández (2010) considera que el propósito es describir y analizar las vivencias cotidianas de las personas un contexto determinado; así como los significados que le dan a ese comportamiento realizado bajo circunstancias comunes o especiales. En este proceso investigativo, el método etnográfico permitió describir y comprender a la luz de las políticas de inclusión educativa y las prácticas pedagógicas de los docentes, cómo se concreta el aprendizaje y socialización de los niños con algún diagnóstico.

La población objeto de estudio la constituyen veinte centros educativos de primera infancia del área metropolitana de Medellín, con niños de 5 a 7 años de edad y que presenten algún tipo de diagnóstico en dificultades para el aprendizaje, sea de tipo sensorial, cognitivo o psicomotriz; $y$ las docentes que se desempeñan en estos centros. La muestra es por conveniencia, corresponde a 30 docentes del grado de preescolar y primero, que autorizaron indagar sobre las prácticas pedagógicas que realizan en su quehacer educativo.

\section{Análisis y resultados}

\section{I. El diseño Universal de Aprendizaje - DUA}

Luego de un proceso de rastreo, recolección y sistematización de información se pudo determinar que para hablar de prácticas pedagógicas es necesario reconocer que los conceptos de aprendizaje han variado con el tiempo, de esta manera se podría decir que "La meta de la educación en el siglo XXI no es simplemente el dominio del conocimiento. Es el dominio de del aprendizaje" CAST (2008) y en este orden de ideas, el DUA pretende que la educación transforme aprendices en aprendices expertos que aprenden $y$ se motivan para aprender, que, a su manera, están preparados para una vida de aprendizaje duradero a lo largo de sus vidas. Dado lo anterior, el DUA pretende fomentar unos procesos pedagógicos accesibles a todos a través de la flexibilización del currículo para que se ajuste a las necesidades y los ritmos de todos, de tal manera que no existan desventajas para los más vulnerables y las barreras se puedan superar fácilmente. También pretende que el reto de la diversidad sea superado a través de metodologías flexibles y el diseño de estrategias para atender y reconocer las múltiples necesidades educativas del mayor número de usuarios (Alba, 20I2)
Este diseño de aprendizaje presenta tres estilos de aprendizajes: Auditivo, Kinestésico y visual, y tres principios necesarios en los diferentes procesos de aprendizaje:

- Múltiples medios de representación: el "iqué?" del aprendizaje.

- Múltiples medios de expresión: el "icómo?" del aprendizaje.

- Múltiples medios de compromiso: el "ipor qué?" del aprendizaje.

Bajo estos principios, el DUA propone prácticas de enseñanza inclusivas donde no es necesario separar los contenidos, o planear diferente y menos separar por grupos de discapacidad o alumnos regulares, que las didácticas pedagógicas de aula se planeen y ejecuten en tal dinamismo que haya lugar a los principios mencionados y por lo tanto los participantes puedan participar de acuerdo a sus capacidades y fortalezas. En este sentido, el currículo se define en líneas generales con "cuatro componentes básicos: I. Metas: Los puntos de referencia $\circ$ expectativas para el aprendizaje y la enseñanza. A menudo se hacen explícitos en la forma de competencias o habilidades a alcanzar 2. Métodos: Los métodos instructivos específicos para el profesor, 3. Materiales: Los medios y los materiales que son utilizados para enseñar y aprender. 4. Evaluación: Las razones y los métodos que se usan para valorar el progreso de los alumnos" CAST (2008. p.7). Con lo anterior queda claro que el docente siempre será el mediador entre un currículo incluyente y otro excluyente, será la dinámica creativa de una pedagogía en movimiento la que establezca los límites de la inclusión.

\subsection{Ejercicios pedagógicos en el DUA (Por Dispositivos básicos de aprendizaje)}

\section{Atención y Concentración}

- Utilizar un lenguaje claro y preciso.

- Tener menos dispersores en aula (móviles, ruidos, ventanales externos).

- Utilizar actividades variadas en cada momento: evitan la fatiga y el aburrimiento.

- Recordarle varias veces sus deberes, adecuar preguntas de seguimiento para volver a atraer su atención.

- Es importante que la actividad no sea demasiado fácil, ayudar sólo cuando sea necesario.

- Mantener el hilo del tema (coherencia).

- Permita la participación activa del estudiante.

- Tomar notas en lugar de transcribir. (hacer relatorías, diarios de campo).

- Haga preguntas relativas al tema a sus estudiantes.

- Pausas activas de 2-3-4 minutos, (juegos, ejercicios corporales) haga que todos participen.

\section{Motivación y la memoria}

- Explique el propósito del aprendizaje. Contextualice la enseñanza. EL QUÉ

- Utilice la mayor cantidad de recursos (visuales, táctiles.... Musicales). No es decir muchas cosas en las clases, sino, decir el mismo tema de diferentes maneras o enfoques.

- Utilice una agenda como guía para despertar el interés de los 
Prácticas pedagógicas inclusivas desde el diseño universal de aprendizaje y plan individual de ajuste razonable temas desde el principio hasta el final de la clase.

- En lo posible realice actividades prácticas donde los estudiantes puedan crear y recrear su aprendizaje.

- Existen estrategias tanto para evocar, precalentamiento del aprendizaje (warm up) conocimientos previos: artefactos exteriores, fichas nemotécnicas, juegos de palabras, rompecabezas temáticos, juego de roles...

\section{Habituación}

- Diálogo constante con los padres o acudientes y con el estudiante con miras a mantener rutinas, pautas y métodos de estudio, a modo de formación.

- Trabajo de exposición en grupos, que contenga al menos 3 de los dispositivos básicos de aprendizaje.

- El docente deberá presentar siempre su agenda para cada sesión de tal manera que los estudiantes tengan visualizados los pasos a seguir.

- El uso de un cuaderno comunicador o agenda personal ayudará a mantener la concentración y facilitará a la familia el acompañamiento.

- Elijan un representante que socialice al resto del grupo.

- La familia habilitará espacios propicios y tiempos permanentes para el seguimiento pedagógico en el hogar.

La inclusión debe generar tal bienestar en los individuos hasta el punto en que "deberán ser capaces de vivir una vida humana de duración normal hasta su fin, sin morir prematuramente o antes de que la vida se reduzca a algo que no merezca la pena vivir" (Nussbaum, 2012.p.49). ¿Pero quién es el encargado del éxito? El maestro a través de su arma más poderosa: "la pedagogía como "la forma en que se evidencia la práctica del docente, que debe ser de forma intencionada, las acciones deben ser justificadas, e influyen varios aspectos donde se desarrolla la acción pedagógica y del contexto, por lo que la pedagogía se aplica por medio de uno o varios métodos”. (Martínez, M. p. I3 I)

\subsection{Prácticas de aula}

Se propone un trabajo interdisciplinario: directivos docentes, psicólogos, docente de aula de apoyo, cada uno desde su experiencia aporta. Aquí algunas sugerencias para una clase inclusiva desde diferentes áreas:

I. Autoconfianza: cree un ambiente de confianza, sensibilizándolos acerca de los diferentes ritmos de aprendizaje, de personalidad y de intereses en los aprendices.

2. Motivación: los primeros encuentros docente - estudiante son fundamentales, propicie actividades que hagan ver el acceso al conocimiento como un asunto de fácil comprensión. Valore lo que aporta con frases como "está bien, pero hay que hacer...; esta parte esta correcta, hay que mejorar la otra; si lees mejor puedes encontrar la respuesta; si necesitas mayor explicación, me llamas; creo que lograras hacerlo, si te concentras,

...". Expresiones como: "Hoy estuviste mejor, concéntrate, cree en ti mismo y hazlo; eres capaz; y gástate el tiempo que necesites..."

3. Núcleos de interés: es necesario hacer una pesquisa que evidencie los focos de interés y mantener el registro en una ficha como la siguiente:

Tabla I: Ficha de caracterización estudiantes NEE

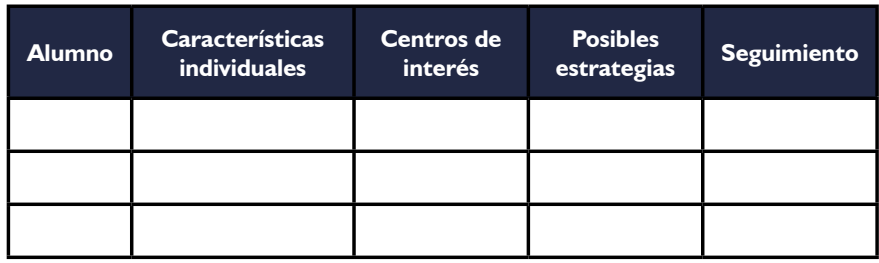

Elaboración de los autores

4. Darle protagonismo, y renombre dentro del grupo, desígnele funciones.

5. Comunicación efectiva y asertiva: sugerir una agenda de tareas actualizada, con tareas, investigaciones o actividades extras. Comunicación permanente con su acudiente y seguimiento al acompañamiento familiar.

6. Reglas de clase visibles: las aulas que tienen niños con barreras para el aprendizaje deberán construir y publicar de manera visible, así recordará su compromiso.

7. Pausas activas: actividades de corta duración, 2-5 minutos: ejercicios motrices, de relajación, canciones que se la sepan, desplazamiento fuera del aula, juego de roles, trabalenguas, un chiste, entre muchos más.

8. Material didáctico: use diferentes fuentes, desde las TICs hasta juegos de mesa. Los materiales visibles son muy efectivos excepto si es una discapacidad visual este caso serán táctiles.

9. Trabajo por proyectos: la dinámica de cada área es de autonomía de los docentes, pero el trabajo por proyectos centra la atención en varios frentes como la investigación desde los mismos núcleos de interés y contribuyen a su motivación, protagonismo y salida de la clase magistral, monótona e improductiva.

10. El trabajo cooperativo: este nivel de intervención produce en ellos confianza y la sensibilización de los demás compañeros. El grupo que lo acoja deberá ser consciente de que no es tenerlo como miembro pasivo, que deberán darle un papel dentro del trabajo, y que el grado de dificultad o responsabilidad empieza por ser menor hasta que el estudiante con barreras a suma un rol responsable.

\section{Plan integrado de ajustes razonables (PIAR)}

\section{I. Aspectos legales}

El plan integrado de ajustes razonables nace en el año 2017 con la promulgación del Decreto I42I y que direcciona las acciones educativas de inclusión para que los centros educativos y los docentes usen mejores herramientas para la flexibilización curricular en estudiantes que tengan dificultades de aprendizaje. Este permite determinar los apoyos pedagógicos para cada estudiante, en función de las necesidades educativas surgidas de la caracterización hecha desde las primeras fases del proceso de inclusión y que se pueda garantizar el éxito escolar donde no haya cabida al fracaso (MEN. 2010)

\subsection{Formulación de los PIAR}

Al respecto, cada institución educativa deberá diseñar un formato para su implementación y teniendo en cuenta modificaciones: 
- En la realización de las actividades.

- En los materiales.

- En los espacios

- Apoyos para facilitar su comunicación.

- Apoyos humanos para el proceso de aprendizaje: Intérpretes, especialistas de la salud y otros.

- Ayudas tecnologías para favorecer el acceso a la información y aprendizaje.

\section{Así mismo deberá dirigirse a conocer y a actuar sobre:}

- Contexto y vida familiar.

- Habilidades intelectuales

- Bienestar emocional.

- Conducta adaptativa y desarrollo personal.

- Salud y bienestar físico.

- Participación e inclusión social.

- Metas de aprendizaje.

Para llevar a cabo una buena planeación de los PIAR debe tenerse una adecuada caracterización de la población y de las implicaciones de la flexibilización curricular, para lo cual se recomiendan tres etapas:

I. Etapa de identificación: se realiza por parte del padre - del profesor cuando observa un desempeño académico y/o comportamental diferente a los demás estudiantes y que interfiere en su desempeño personal, y en ocasiones en el de los demás compañeros de clase. También es importante una valoración del desarrollo de las dimensiones de desarrollo y de las competencias que tiene el estudiante con la cual se solicita una evaluación integral por los profesionales de salud

5. Etapa de valoración y diagnóstico: el padre de familia debe garantizar que su hijo sea valorado integralmente $y$ dar a conocer el diagnóstico a la Institución. Esta es una de las barreras más grandes, la aceptación de la familia que su hijo tienen unas características diferentes para acceder al aprendizaje y que requerirá de mayor acompañamiento (Figueroa, 2017).

6. Etapa de apoyo institucional y familiar: el diagnóstico debe darse a conocer a los profesores y al servicio de profesional de apoyo. Se inician las adaptaciones en los procesos de valoración y evaluación del niño. Si no se cuenta con el diagnóstico, o mientras se conoce, la institución debe hacer una caracterización del estudiante, en sus aspectos familiares, sociales y de desempeño básico en acciones y conocimientos escolares básicos.

\section{CONCLUSIONES}

La práctica pedagógica se entiende como el espacio de interacción y reflexión en torno a problemas, saberes, vivencias y aprendizaje de los sujetos en formación, son todos aquellos elementos dinamizadores y transformadores del acontecer diario del docente (Cobos, 2000). En otras palabras, constituyen el quehacer máximo del maestro en un contexto socio cultural, pero que debe ser tan cercano al ser humano que el disfrute sea una constante para ambos participantes. En el trabajo descrito, prevalecen las acciones pedagógicas propuestas y registradas durante la investigación en la cual se trató de sistematizar las diferentes prácticas que pueden resultar de una aplicación permanente y adecuada del DUA aplicado en los PIAR.

Las prácticas pedagógicas son la clave en el desarrollo de las capacidades de los niños y jóvenes que están en situación diversa de aprendizaje, pero carecerían de valor académico si la familia, aún con las intenciones de dar una adecuada educación a sus hijos, no tienen la voluntad plena y comprometida con la escuela para utilizar las diferentes herramientas que le da la Ley - la academia para enfrentarse al reto de formar seres humanos integrales y con oportunidades de desarrollo a nivel social y personal (UNICEF, 20I4). Adicional a lo anterior, es necesario reflexionar en lo que plantea Melero "En una sociedad democrática, el dar respuestas a la diversidad no consiste en proponer programas específicos, sino en erradicar la exclusión. (2016.p. 166).

Los objetivos latentes en la utilización del DUA y los PIAR son buscar el desarrollo personal desde estrategias donde el estudiante reconstruya su autoestima, como ser con potencialidades para..., que sea capaz de desempeñarse con eficiencia en muchas tareas que le exige la construcción de conocimiento. Un segundo objetivo consiste en construir seguridad en sí mismo, mostrársele que puede llegar a sus metas y que tiene potencialidades para hacerlo, aunque requiera mayores esfuerzos que otros. Como tercer objetivo se plantea que las interacciones sociales son un eje fundamental en los procesos de inclusión y que a través de las prácticas descritas puedan reconocerse como individuos y miembros de un grupo, compartir, tener amigos, rivales; aprender a negociar, a ganar $y$ a perder. Por último, el compromiso y esfuerzo requerido pata el aprendizaje involucra a la traída familia, escuela y estudiante.

En los aspectos de Evaluación y Promoción, según el diseño de los PIAR, se contemplan todas las dificultades que comprometen algún nivel o facultad de su proceso y en las cuales se deben tener en cuenta aprendizajes básicos como:

- Dominio del lenguaje oral y escrito y otras formas de comunicación.

- Manejo de las matemáticas básicas: problemas cotidianos.

- Manejo corporal para ubicación en el espacio.

- Arte y creatividad para expresarse.

- Uso de las técnicas básicas de la información y la comunicación.

- 6. Relaciones interpersonales, interculturales y sociales.

- Autonomía y emprendimiento.

El registro de sus logros debe ser descriptivo - cualitativo, y se basará en lo que sabe y puede hacer, estableciendo niveles de desarrollo en una escala progresiva donde se valoran los logros mínimos teniendo en cuenta que los tiempos de estos niños son distintos a los tiempos escolares establecidos para la población regular

La siguiente tabla intenta mostrar algunos ejemplos de descriptores básicos y niveles para pensar la promoción de un grado a otro (describe lo que sabe hacer, I BAJO, 2, MEDIO, 3 BIEN). 
Aprendo aspectos sobre el medio ambiente, datos históricos, sociales. .. (conocimientos básicos)

Utilizo el habla como una herramienta para comunicarme con otros.

Utilizo la lectura para interpretar temas de ciencias, las sociales...literatura (cuentos entre otros)

Utilizo la escritura para expresar mis ideas respecto a un tema (narro, describo, argumento) en situaciones concretas.

Participo en todas las actividades propuestas en las clases. 


\section{Referencias bibliográficas}

Alba Pastor, C. (20I2). Aportaciones del Diseño Universal para el Aprendizaje y de los materiales digitales en el logro de una enseñanza accesible, en Navarro, J., Fernández, Ma T., Soto, F. J.

American Association on Intellectual and Developmental Disabilities (aaidd) (2012). Discapacidad intelectual: definición, clasificación

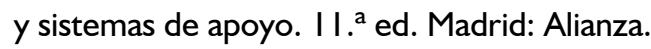

Arnaiz, S. P. (20I I). Escuelas eficaces e inclusivas: Cómo favorecer su desarrollo. Educatio Siglo XXI, 27.

Arnaiz, S. P. (2003). Educación Inclusiva: Una escuela para todos. Aljibe, España.

Blanco, R. (20II) “De la inclusión en la escuela a la Inclusión en el aprendizaje”, Palabra Maestra, Premio Compartir al Maestro, año I I. Número 28, Bogotá, Colombia.

Bonilla. E, Rodríguez, P (2005). Más allá del dilema de los métodos: la investigación en ciencias sociales, editorial Norma.

CAST (2008). Universal design for learning guidelines version I.0. Wakefield, MA.

Castejón y Navas. (1990). Unas Bases OPsicológicas de la Educación Especial. Alicante: Editorial Club Universitario.

Congreso de la república de Colombia, Ley II 5 de 1994: “Ley General de Educación”. Recuperado de http://www.alcaldiabogota. gov.co/sisjur/normas/Normal .jsp?i=292

Congreso de la república de Colombia, 2013, Ley Estatutaria 1618 Inclusión educativa.

De Soussa, (2010) Descolonizar el saber, reinventar el poder. Ediciones Trilce. Uruguay.

Di Nasso, Patricia. Mirada Histórica de la Discapacidad. Patricia Di Nasso. Fundació Cátedra lberoamericana. Universitat de les Illes Balears. Fuente: http://fci.uib.es/Servicios/libros/articulos/ di_nasso/.

Eglér Mantoan, M. (2017). Educación especial en la perspectiva inclusiva: lo que dicen los profesores, directores y el país (Special education in the inclusive perspective: what teachers, directors and the country say). Inclusión Y Desarrollo, 5(I), 7-22. https://doi. org/10.26620/uniminuto.inclusion.5.1.2018.7-22.

Eglér Mantoan, M. (2017). Educación especial en la perspectiva inclusiva: lo que dicen los profesores, directores y el país (Special education in the inclusive perspective: what teachers, directors and the country say). Inclusión Y Desarrollo, 5(I), 7-22. https://doi. org/10.26620/uniminuto.inclusion.5.1.2018.7-22

Figueroa, L. A. (2017). Ley de Inclusión Educativa: Impacto en la ciudad de Medellín. Horizontes Pedagógicos, issn-I:0123-8264, I9 (2), I0I-[108. Obtenido de: https://revistas.iberoamericana.edu.co/index.php/rhpedagogicos/ article/view/I2I0

Gimeno, J. (2004): Quién fracasa cuando hay fracaso escolar? En: I Congreso anual sobre el fracaso escolar. Palma de Mallorca. En:http://www.fracasoescolar.com/congrescat/conclusions2004.html. (Recuperado en: 5 de noviembre de 20l4).

Gimeno, J. (2006). La construcción del discurso acerca de la diversidad y sus prácticas. En, WAA: Atención a la Diversidad. Barcelona: Graó.

Gough.I. (2007) El enfoque de las capacidades de M. Nussbaum: un análisis comparado con nuestra teoría de las necesidades humanas. Publicado en: Papeles de Relaciones Ecosociales y Cambio Global, n 100, CIP-Ecosocial/lcaria, invierno $2007 / 08$.

Gutiérrez, I. (1997). Introducción a la historia de la logopedia. Madrid: Narcea, S.A. De ediciones.

Hernández, Elsa. Desarrollo Histórico de la Discapacidad. Evolución y Tratamiento. Elsa Hernández. Fuente: http://www.iin.oea.org/ Cursos_a_distancia/cad_guia_disc_UTI.pdf.

Hernández Sampieri, Roberto; Fernández Collado Carlos Baptista Lucio, Pilar (2010). Metodología de la investigación. México: McGraw-Hill Interamericana.

López, Melero, M. (201 I). Barreras que impiden la escuela inclusiva y algunas estrategias para construir una escuela sin exclusiones. En: http://www.doe.uma.es/repository/fileDownloader?rfname=43862855-35b3- 40f2-84e6-ecdf27f9307b.pdf (Recuperado el 20 noviembre de 2013).

López, Melero, M. (2016). La LOMCE, ( a dismal picture that fails to respond to diversity) un cuadro tenebroso que no responde a 
Marchesi, A. (1987). El desarrollo cognitivo y lingüístico de los niños sordos. Madrid: Alizanza.

Martínez C.M. (20I5). Los fundamentos pedagógicos que sustenta la práctica docente de los profesores que ingresan y egresan de la maestría en intervención socioeducativa. Revista Universia, No I5. V. 6. https://ac.els-cdn.com/S20072872I530007X/Is2.0-S20072872I530007X-main.pdf?_tid = I9a0880e-d74e-4dea-88e6-cl 579369960I\&acdnat =I543I90478_ cf0c886c I fafea55804bceb l 6ed05456

MEN (2010). Educación de calidad: el camino para la prosperidad. Recuperado el 7 de abril de 2014 de http://www.mineducacion. gov.co/cvn/1665/articles-237397_archivo_pdf.pd.

Muntaner G.J. (20I3) Calidad de vida en la escuela inclusiva. Revista Iberoamericana De Educación. N. 63 (20I3), pp. $35-49$ (I0226508) - OEI/CAEU

Nasbaum, M. (20I I). Creating Capabilities. The Human Develpment Approach. Cambridge: The Belknap Press of Harvard University Press.

Nussbaum, M. (20I2). Crear capacidades: Propuesta para el desarrollo humano. Barcelona: Paidós.

Ocampo González, A. (20I5). Perspectivas para un "enfoque re-construccionista» de la "educación inclusiva» en el siglo $X X I$ (Prospects for a "Reconstructionist Approach" of "Inclusive Education\&quot; in the XXI century). Inclusión Y Desarrollo, 3(I), 3450. https://doi.org/10.26620/uniminuto.inclusion.3.1.2016.34-50

ONU, 1948, Declaración Universal de los Derechos Humanos,

ONU, 2008/2009, Universidad de los Andes en Asamblea General de la ONU Resolución 3447 (XXX), 09 de diciembre de I975 Declaración Universal de los Derechos del Impedido.

Salmi, J. (2016). Políticas económicas internacionales para el desarrollo de la inclusión educativa en Colombia desde la perspectiva de Jamil Salmi (International economic policies for development of educational inclusion in Colombia from the perspective of Jamil Salmi). Inclusión Y Desarrollo, 4(I), I4-16. https://doi.org/10.26620/uniminuto.inclusion.4.1.2017.14-16

UNESCO (2007). Discusión de las políticas educativas para América Latina y el Caribe. (2007). Educación de calidad para todos. En: http://unesdoc.unesco.org/images/00 I 5/001502/I50272s.pdf (Recuperado en: I 5 de febrero de 2014).

UNESCO (2008), Educación Inclusiva: El Camino Hacia El Futuro. Centro Internacional de Conferencias, Ginebra. En: http://www. ibe.unesco.org/fileadmin/user_upload/Policy_Dialogue/48th_ICE/CONFINT ED_48_Inf_2_Spanish.pdf (Recuperado en: I0 de mayo de 2014).

UNICEF (20I4). Repitencia: se trabaja sobre la inclusión con nuevas pedagogías. En: http://misionesonline.net/20 I 4/09/I3/repitenciaescolar-una-consecuencia-que-afecta-loschicos-mas-pobres/ (Recuperado en 25 de enero de 20I5).

Viáfara, L. C. (2008b). Cambio estructural y estratificación social entre grupos raciales en la ciudad de Cali - Colombia». (Univalle, Ed.) sociedad y economía(I5), 103-122. Recuperado el 2015.

Warnock, H. y. (1978). Special Educational Needs Report of comity of enquiry into the education of handicapped children and young people. (S. Cero, Ed.) 12-24.

Zaccagnini, M. (2008). Impacto de los paradigmas pedagógicos históricos en las prácticas educativas contemporáneas. Revista Iberoamérica de educación ISSN: 168I- 5653. Recuperado 20 de octubre de 2013 de http://www.rieoei.org/ deloslectores/443Zaccagnini.pdf 